Meredith Bradley

Case Western Reserve University School of Medicine, Cleveland, $\mathrm{OH}$
Praveen Chahar, MD, FCARCSI

Anesthesiology Institute, Cleveland Clinic

\title{
Burnout of healthcare providers during COVID-19
}

\section{Posted June 23, 2020}

\section{ABSTRACT}

Physician burnout is not new, but the COVID-19 pandemic is accelerating the many negative repercussions of uncertainty and inadequate support, and the consequences are being felt by patients, physicians, and healthcare systems.

\section{BACKGROUND}

Physician burnout has serious consequences to the individual physician, to patients, and to healthcare institutions. Research has shown the prevalence of burnout to be more than $40 \%$, with highest rates in frontline healthcare providers such as emergency medicine, primary care, and critical care. ${ }^{1}$ COVID-19 presents new stressors for healthcare providers, and recent events involving self-harm by physicians have brought increased attention to the emotional impacts of caring for these critically ill patients. ${ }^{2}$

\section{NEW STRESSORS IN COVID-19}

COVID-19 presents multiple stressors to healthcare providers, including risk of infection, social isolation, and economic consequences. The practice of medicine has changed with COVID-19, with decreasing outpatient revenue, reductions in salary and benefits, and increased use of telemedicine, with its effect on the doctor-patient relationship.

A survey by the Society of Critical Care Medicine of nearly 9,500 critical care providers showed that median self-reported provider stress has increased from a score of 3 to a score of $8 .{ }^{3}$ Top stressors included lack of personal protective equipment, fear of contracting COVID-19, and fear of spreading the infection to family members. ${ }^{3}$ These work-related stressors have been associated with anxiety and depression, and

The statements and opinions expressed in COVID-19 Curbside Consults are based on experience and the available literature as of the date posted. While we try to regularly update this content, any offered recommendations cannot be substituted for the clinical judgment of clinicians caring for individual patients.

doi:10.3949/ccjm.87a.ccc051 they reflect real physical and emotional risks faced by frontline providers. ${ }^{4}$ In addition to these concerns, another recent survey also noted worries about being asked to care for patients more critically ill than they are trained for and about the limited availability of up-to-date information. ${ }^{5}$

Research out of China about mental health outcomes of healthcare workers treating COVID-19, although limited, indicates that nurses, women, and frontline healthcare workers experience the highest psychological burden and develop the most unfavorable mental health outcomes, including depression, anxiety, and insomnia. ${ }^{6}$

Social and family support are vital pillars in promoting resilience and preventing burnout; however, COVID-19 poses challenges to both. ${ }^{7}$ Healthcare providers are hesitant to spend time with family members due to the risk of spreading infection to their loved ones, and many healthcare providers isolate themselves within their homes. Similarly, social distancing makes it more challenging to keep in touch with friends.

Many providers are experiencing increased demands at home, as the closing of schools and daycare centers has made finding childcare a major challenge, particularly when the healthcare provider is a single parents or when both parents are essential employees. ${ }^{5}$ In addition, childcare workers are hesitant to work in homes of healthcare workers due to fear of getting COVID-19.

COVID-19 is presenting economic challenges, with decreased revenue in hospitals and outpatient practices. Decreasing revenues are leading to reductions in salary, benefits, and vacation time to make up for lost revenue.

\section{SIGNS OF BURNOUT}

Burnout does not have a universal definition and is specifically job-related, as opposed to depression. ${ }^{1,4}$ Common signs of burnout are feelings of emotional exhaustion, cynicism, depersonalization, and low 


\section{TABLE 1}

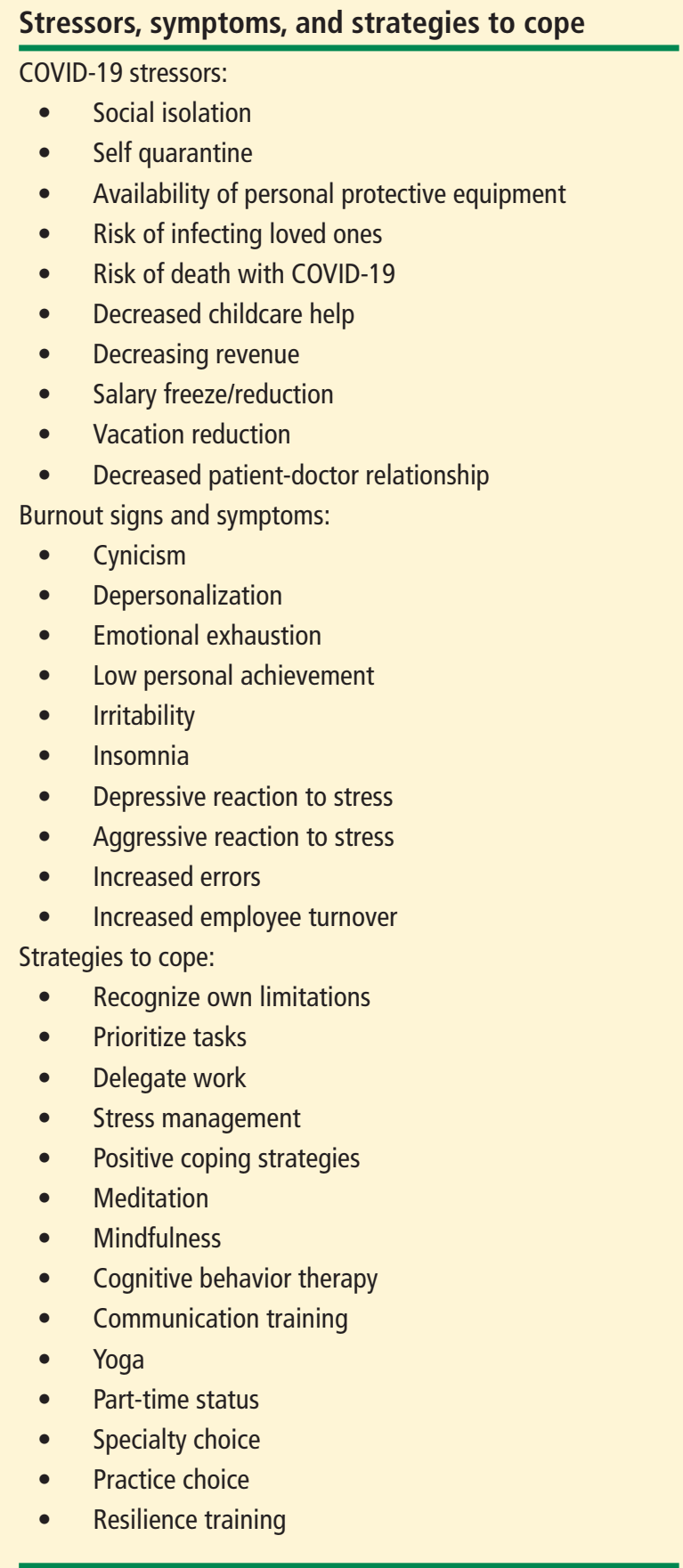

personal accomplishment. Maslach Burnout Inventory, the gold standard for measuring occupational burnout, is a 22 -item questionnaire measuring three domains of burnout: emotional exhaustion, depersonalization, and low personal achievement. A high score on emotional exhaustion and depersonalization and a low score on personal accomplishment indicates burnout. And the response to even single statements such as "I feel burned out from my work" and "I've become more callous toward people since I started this job" have shown good correlation with emotional exhaustion and depersonalization. ${ }^{8}$

\section{CONSEQUENCES OF BURNOUT}

Physician burnout has negative consequences to the physician, patients, and the organization. It is associated with increased rates of medical error and decreased productivity, putting patients at risk and straining an already overstretched medical system. ${ }^{1}$ Perhaps most pressing now are the well-studied links between physician burnout and depression, alcohol abuse, and suicidal ideation. It is well documented that physicians take their own lives at rates much higher than the general public, and on average, 400 US physicians die by suicide each year. ${ }^{4}$ Drastically increasing stressors on healthcare providers both during work and outside of work without adequate approaches for how to combat these stressors will increase the rate of physician burnout.

\section{HOW TO PREVENT BURNOUT DURING A PANDEMIC}

Preventing burnout relies on interventions at individual, team, and institutional levels. Research has shown benefits of mindfulness, stress-management training, exercise programs, and participation in small-group programs focusing on community, connectedness, and meaning in helping providers deal with burnout. ${ }^{1,6}$ Identifying signs of burnout in friends and colleagues and reaching out can be an important first step in getting them support (Table 1).

Organizational strategies such as limiting physician hours, particularly in intensive care units, promoting a sense of community and core values, and engaging physicians in establishment of new protocols has been shown to prevent burnout. ${ }^{1,5}$ Ensuring availability of personal protective equipment and being open with staff about current resources can ease some of the anxiety that comes from uncertainty. Providing accurate and timely information about providing care to COVID-19 patients can promote standardization and encourage efficiency throughout the healthcare system..$^{5,9,10}$

There have also been team-based strategies adapted from those used in the military, where groups are routinely asked to work in high-stress environments. ${ }^{11}$ Having end-of-shift debriefing sessions normalizes talking about anxiety and grief and gives people tools 
to voice their experiences. Having "battle buddies," people paired with a partner to support and check in on one another, can provide support in a way that friends and loved ones who are not in healthcare may not be able to do.

\section{CONCLUSION}

The mental health impacts of COVID-19 on frontline healthcare workers are undeniable. While physician burnout is not new, the pandemic is rapidly accelerating the many negative repercussions of uncertainty and inadequate support, and the consequences are being felt by patients, physicians, and healthcare systems. However, this time of change is an opportunity for a cultural shift in how we perceive and manage physician mental health. Perhaps the tragic events involving self-harm in New York City can serve as a wake-up call to the emotional tolls of caring for COVID-19 patients and our responsibility to support ourselves and our colleagues.

\section{TO GET HELP}

If you or someone you know is having thoughts of suicide, contact the National Suicide Prevention Lifeline at 1-800-273-8255 (TALK), or text the Crisis Text Line by texting "4Hope" to 741741.

The Federation of State Physician Health Programs can help provide confidential resources for healthcare providers affected by substance-related and addictive disorders, mental illness, and other illness. https://www.fsphp.org/state-programs

\section{REFERENCES}

1. West CP, Dyrbye LN, Shanafelt TD. Physician burnout: contributors, consequences and solutions. J Intern Med 2018; 283(6):516-529. doi:10.1111/joim.12752

2. Watkins A, Rothfeld M, Rashbaum WK, Rosenthal BM. Top E.R. doctor who treated virus patients dies by suicide. The New York Times. https://www.nytimes.com/2020/04/27/nyregion/new-yorkcity-doctor-suicide-coronavirus.html. Accessed June 22, 2020.

3. Society of Critical Care Medicine. Clinicians report high stress in COVID-19 response. https://sccm.org/Blog/May-2020/SCCM-COVID19-Rapid-Cycle-Survey-2-Report. Accessed June 22, 2020.

4. Stehman CR, Testo Z, Gershaw RS, Kellogg AR. Burnout, drop out, suicide: physician loss in emergency medicine, Part I. West J Emerg Med 2019; 20(3):485-494. doi:10.5811/westjem.2019.4.40970

5. Shanafelt T, Ripp J, Trockel M. Understanding and addressing sources of anxiety among health care professionals during the COVID-19 pandemic. JAMA 2020; Apr 7. doi:10.1001/jama.2020.5893

6. Lai J, Ma S, Wang Y, et al. Factors associated with mental health outcomes among health care workers exposed to coronavirus disease 2019. JAMA Netw Open 2020; Mar 2. doi:10.1001/ jamanetworkopen.2020.3976

7. Sasangohar F, Jones SL, Masud FN, Vahidy FS, Kash BA. Provider burnout and fatigue during the COVID-19 pandemic: lessons learned from a high-volume intensive care unit. Anesth Analg 2020; 131(1):106-111. doi:10.1213/ANE.0000000000004866

8. West CP, Dyrbye LN, Sloan JA, Shanafelt TD. Single item measures of emotional exhaustion and depersonalization are useful for assessing burnout in medical professionals. J Gen Intern Med 2009; 24(12):1318-1321. doi:10.1007/s11606-009-1129-z

9. Zhang $\mathbf{H}, \mathbf{Y e} \mathbf{Z}$, Tang $\mathbf{L}$, et al. Anxiety symptoms and burnout among Chinese medical staff of intensive care unit: the moderating effect of social support. BMC Psychiatry 2020; 20(1):197. doi:10.1186/ s12888-020-02603-2

10. Adams JG, Walls RM. Supporting the health care workforce during the COVID-19 global epidemic. JAMA 2020; March 12. doi:10.1001/ jama.2020.3972

11. Smith RE. Lessons from a different war for preventing moral injury in clinicians. STAT. https://www.statnews.com/2020/04/01/lessons-different-war-protecting-clinicians-moral-injury/. Accessed June 22, 2020.

Correspondence: Praveen Chahar, MD, FCARCSI, Anesthesiology Institute, NA23, Cleveland Clinic, 9500 Euclid Avenue, Cleveland, $\mathrm{OH} 44195$; chaharp@ccf.org 